\title{
Deep ultraviolet photoluminescence studies of AIN photonic crystals
}

\author{
N. Nepal, J. Shakya, M. L. Nakarmi, J. Y. Lin, and H. X. Jiang ${ }^{\text {a) }}$ \\ Department of Physics, Kansas State University, Manhattan, Kansas 66506-2601
}

(Received 1 December 2005; accepted 12 February 2006; published online 29 March 2006)

\begin{abstract}
Two-dimensional AIN photonic crystals (PCs) with varying periodicity/diameter down to $150 / 75 \mathrm{~nm}$ were fabricated. Deep ultraviolet photoluminescence spectroscopy has been employed to study the optical properties of AlN PCs. With PC formation, a 20-fold enhancement in the band edge emission intensity at $208 \mathrm{~nm}$ over unpatterned AlN epilayer has been observed. The emission intensity increases with decreasing lattice constant of AIN PCs. However, the spectral peak energy decreases with decreasing lattice constant, indicating a possible release of compressive stresses as a result of PC formation. Successful fabrication of AIN PCs opens up new opportunities for exploring novel physical phenomena in the artificially structured photonic band gap material system and their applications, particularly in the area of deep UV photonics. (C) 2006 American Institute of Physics. [DOI: 10.1063/1.2190452]
\end{abstract}

Recently, there has been intensive R\&D effort in the area of ultraviolet (UV) and deep UV optoelectronic materials and devices. One of the main challenges is finding effective methods and schemes to enhance the extraction efficiencies of deep UV emitters. A large fraction of the light generated in the semiconductors is trapped by a total internal reflection as guided modes due to the higher refractive index of semiconductor and eventually lost due to the parasitic absorption within the semiconductor. Moreover, the UV emitters using $\mathrm{AlGaN}$ as active layers possess unique polarization properties compared to more conventional semiconductors. In Alrich $\mathrm{AlGaN}$ alloys the recombination between the conduction band electrons and holes in the top-most valance band is prohibited for the polarization configuration of $\mathbf{E} \perp c$, while the allowed $\mathbf{E} / / c$ emission component cannot easily get out of the escaping cone. ${ }^{1,2}$ Here, $\mathbf{E}$ denotes the electric field component of the light and $c$ the $c$ axis of the epilayers. Various schemes have been employed to enhance the extraction of light from light emitting diodes (LEDs). Among these techniques, surface roughening, ${ }^{3-6} \mu$-LED arrays, ${ }^{7}$ photonic crystals (PCs), ${ }^{8-19}$ and integrated microlens arrays ${ }^{20}$ have been utilized.

Much progress has been made on the understanding and fabrication of PCs that function in the infrared (IR) region. $^{8-11}$ A maximum of 30 -fold enhancement in a photoluminescence $(\mathrm{PL})$ emission intensity from a freestanding InGaAs PC slab has been reported at a wavelength of $1100 \mathrm{~nm} .{ }^{10}$ A six-fold enhancement in emission intensity at $925 \mathrm{~nm}$ has been obtained from an LED structure upon PC formation under optical pumping. ${ }^{11}$ Nitride PCs that function in the visible and UV regions have been studied only very recently due to the challenges associated with the fabrication of PCs with submicron periodicity. ${ }^{12-19}$ We reported a 20fold enhancement in the PL emission intensity from an InGaN/GaN multiple quantum well (MQW) at $475 \mathrm{~nm} .^{12}$ Under the guidance of the optical pumping studies, we have realized the nitride $\mathrm{PC}$ LEDs active in the wavelength range of 333-460 nm and a maximum of 2.5-fold power enhancement at $333 \mathrm{~nm}$ was obtained under current injection. ${ }^{13,15,16}$

\footnotetext{
${ }^{\text {a)} E l e c t r o n i c ~ m a i l: ~ j i a n g @ p h y s . k s u . e d u ~}$
}

In this letter, we report on the successful fabrication and deep UV PL studies of AIN PCs. Two-dimensional arrays of PCs were fabricated on AlN epilayers grown by metalorganic chemical vapor deposition (MOCVD) using electron-beam (e-beam) lithography and inductively coupled plasma (ICP) dry etching. In order to observe the PC effects in the deep UV region, we patterned the air hole size below $100 \mathrm{~nm}$. A 20-fold enhancement in the PL emission intensity in the deep UV region (down to $208 \mathrm{~nm}$ ) was observed upon PC formation in AlN. The emission intensity was observed to increase with a decrease of the PC lattice constant.

The $1 \mu \mathrm{m}$ thick AIN epilayers were grown by MOCVD on sapphire (0001) substrates. Trimethylaluminium (TMAl) and blue ammonia were used as $\mathrm{Al}$ and $\mathrm{N}$ sources, respectively. Atomic force microscopy (AFM) and scanning electron microscopy (SEM) were used to characterize the surface morphology of these epilayers. Structural characterization was done with x-ray diffraction (XRD) measurement. Full width at half maximum (FWHM) of XRD rocking curve of (0002) peak of AlN epilayers was between 50 and 100 arcsec. The PCs with triangular lattice patterns of circular air holes with varying diameter $(d)$ from 75 to $300 \mathrm{~nm}$ and periodicity or lattice constant (a) from 150 to $600 \mathrm{~nm}$ were defined in an area of $10 \times 10 \mu \mathrm{m}^{2}$ using e-beam lithography and ICP dry etching as described previously. ${ }^{12,13,15,16}$ Figures 1(a) and 1(b) show the AFM and SEM images of AlN PCs with periodicity/diameter $(a / d)$ of $180 / 90 \mathrm{~nm}$.

Deep UV PL spectroscopy was employed to investigate the optical properties of AlN PCs. The PL system consists of a frequency quadrupled 100 fs Ti:sapphire laser with an average power of $3 \mathrm{~mW}$ with an excitation photon energy set at $197 \mathrm{~nm}$ or $6.28 \mathrm{eV}$ (repetition rate of $76 \mathrm{MHz}$ ), a monochromator $(1.3 \mathrm{~m}) .^{21}$ An UV objective was used to focus $197 \mathrm{~nm}$ laser beam onto a tight spot of about $2 \mu \mathrm{m}$ in diameter on AlN PCs with different size features. Schematic diagram of the PL setup used for the optical measurement is shown in Fig. 1(c). Samples were mounted on a sample holder in such a way that the $\mathbf{k}$ vector of the emitted light is parallel to the $c$ axis and the incident laser beam with vertical polarization makes $45^{\circ}$ with respect to the $c$ axis.

The room temperature PL spectra of AlN epilayers with and without PC formation are shown in Fig. 2. The dominant 


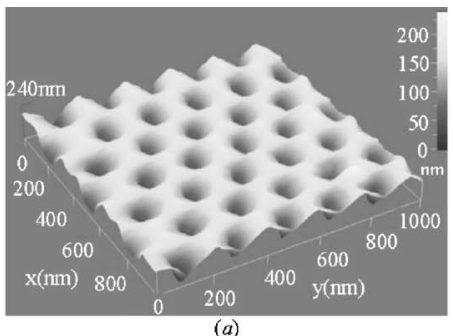

(a)

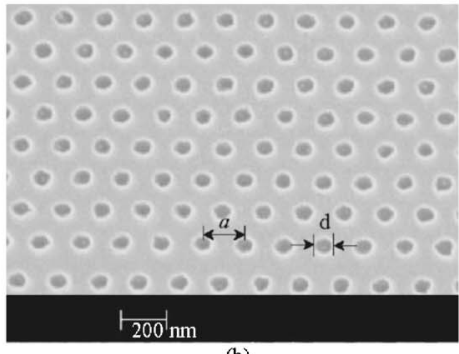

(b)

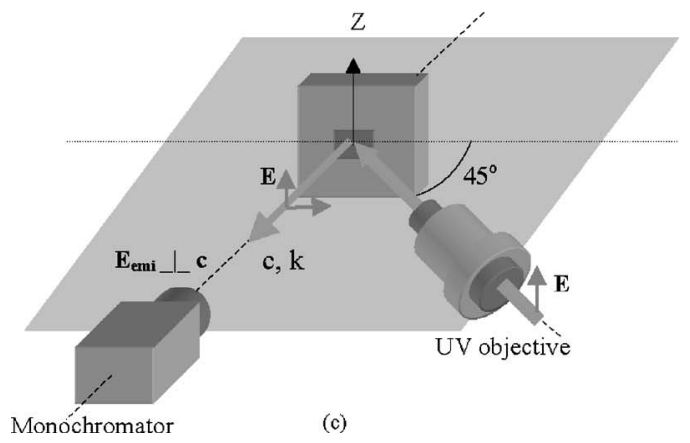

FIG. 1. (a) AFM and (b) SEM images of the AlN PCs with periodicity/ diameter $(a / d)$ of $180 / 90 \mathrm{~nm}$. (c) Schematic diagram of the PL setup for the optical measurement of AlN PCs.

emission line at $5.97 \mathrm{eV}$ is attributed to the band edge emission in AlN. An additional weak emission line indicated as $E_{\text {exc }}-2 \mathrm{LO}$ is also visible, which was attributed to the Raman scattering of the excitation laser line with two longitudinal optical (2LO) phonons with a LO phonon energy of $110 \mathrm{meV} .^{1}$ An interesting fact is that $E_{\text {exc }}-1 \mathrm{LO}$ is absent, which may be related to the symmetry properties involved.

The spectral peak position of the band edge emission decreases to $5.96 \mathrm{eV}$ in AlN PCs of dimension a/d $=180 / 90 \mathrm{~nm}$. There are also emission lines at 5.88 and

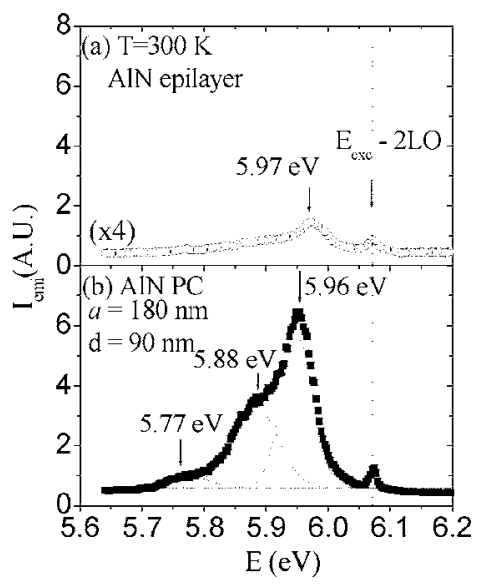

FIG. 2. PL spectra of (a) AlN epilayer (b) AlN PCs with periodicity/ diameter $(a / d)$ of $180 / 90 \mathrm{~nm}$ at $300 \mathrm{~K}$. The PL emission intensity $\left(I_{\mathrm{emi}}\right)$ of AlN PCs is 20 times higher than that of unpatterned AlN epilayer. Downloaded 12 Jul 2010 to 129.118.86.45. Redistribution subject to AlP license or copyright; see http://apl.aip.org/apl/copyright.jsp

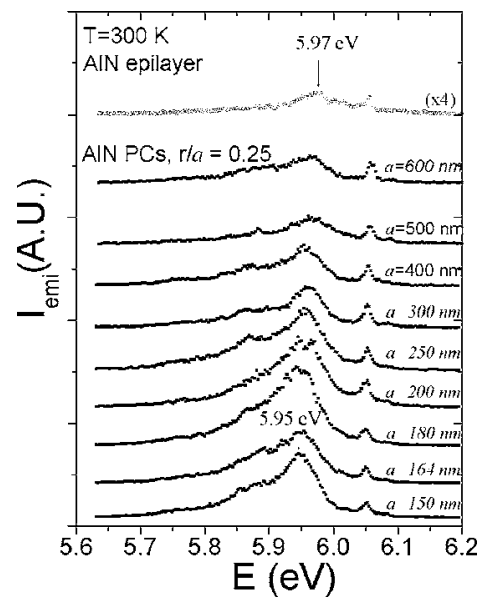

FIG. 3. PC size feature dependence of the PL spectra. The integrated emission intensity $\left(I_{\text {emi }}\right)$ of AlN PCs increases with decreasing lattice constant for a constant air-filling factor $(r / a=0.25)$.

$5.77 \mathrm{eV}$. Since we are probing the sample using vertically polarized laser at an angle of about $45^{\circ}$, mixed $A_{1}-E_{1}$ symmetry phonons may also be observed besides pure transverse optical (TO) or LO phonons. Measured phonon energies of $E_{1}(\mathrm{TO})$ and $A_{1}(\mathrm{LO})$ phonons in AlN were 80 and $110 \mathrm{meV}$, respectively. ${ }^{22}$ We believe that the emission peaks at 5.88 $(80 \mathrm{meV}$ below $5.96 \mathrm{eV})$ and $5.77 \mathrm{eV}(80+110 \mathrm{meV}$ below $5.96 \mathrm{eV})$ are due to the $E_{1}(\mathrm{TO})$ and $E_{1}(\mathrm{TO})+A_{1}(\mathrm{LO})$ phonon replicas, respectively. The formation of PCs on AlN produced emission intensity enhancement of 20 -fold as compared to that of unpatterned AIN epilayer. Besides the band edge transition, PCs also enhance emission intensity of the $\mathrm{TO}$ and $\mathrm{TO}+\mathrm{LO}$ phonon replica lines. PC folds the guided modes at the Brillouin zone boundaries allowing phase matching to the radiation mode that lie above the air light line. Hence enhancement could be due to Bragg scattering of the emitted light by PCs.

Figure 3 shows the periodicity and hole size dependence of the room temperature PL spectra for $a=150-600 \mathrm{~nm}$ measured for AlN PCs. The ratio of air-hole radius $r(d=2 r)$ to the lattice constant $a$ is fixed at 0.25 for all AlN PCs. We attribute the dominant emission line to the band edge transition in AlN PCs and there is a laser 2LO phonon line at $6.06 \mathrm{eV}$ in each spectrum. The spectral peak position of the band edge transition, $E_{p}$, decreases from $5.970 \mathrm{eV}$ at $a=$ infinity (epilayer) to $5.952 \mathrm{eV}$ at $a=150 \mathrm{~nm}$. However, the emission intensity $\left(I_{\mathrm{emi}}\right)$ increases with decreasing the lattice constant.

The lattice constant dependence of the emission peak energy of AlN PCs is shown in Fig. 4(a). The straight line is the linear fit of the data with $E_{p}=E_{p}(a=\infty)-c / a$, where $c$ is a proportionality constant. Figure 4(a) shows that $E_{p}$ decreases linearly with $1 / a$ of PCs. This is due to the fact that the PC feature is small enough to alter the strains in the epilayers. TEM and high-resolution XRD studies provide evidence that the final resultant stress is compressive in our AlN epilayers grown on sapphires. ${ }^{23} E_{p}$ decreases when compressive stresses are released because of etched air holes and the effect is more pronounced for smaller holes. The enhancement factor for $I_{\mathrm{emi}}$ varies linearly with the inverse of the lattice constant $\left(a^{-1}\right)$ of AlN PCs, as illustrated in Fig. 4(b). We define the PL enhancement factor as the integrated PL emission intensity of AIN PCs relative to that of 


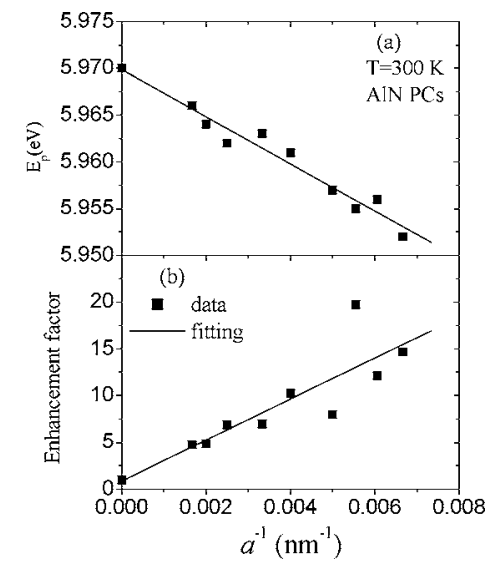

FIG. 4. Lattice constant dependence of the (a) PL emission peak energy and (b) light enhancement factor in AlN PCs. The solid lines are the linear fits of data. The PL emission spectral peak energy decreases, while the integrated emission intensity increases with decreasing lattice constant, $a$.

the unpatterned AlN epilayer. The enhancement factor increases with decreasing lattice constant for a constant airfilling factor $(r / a=$ constant $)$ and reaches a maximum of 20fold for $a=180 \mathrm{~nm}$. Variation of PL enhancement factor with lattice constant has been reported in a previous work at an emission wavelength of $1100 \mathrm{~nm}$ for $\mathrm{InGaAs}_{\mathrm{PCs}}{ }^{10}$ in which the enhancement factor was a maximum at $a=800 \mathrm{~nm}$ and then decreases with $a$ on both sides for either $a>800 \mathrm{~nm}$ or $a<800 \mathrm{~nm}$. In the case of AlN deep UV PCs, the PL enhancement factor increases with decreasing lattice constant in the range of the present study down to $a=150 \mathrm{~nm}$. In a typical as-grown sample, the extraction efficiency is about $5 \%$. Numerically, the 20 -fold enhancement corresponds to an extraction efficiency approaching $100 \%$.

PCs enhance emission intensity in several ways. Enhancement due to Purcell effect may be limited. Bragg scattering of photons by periodically varying refractive indices creates a photon band gap (PBG) in which lateral propagation of the Bloch guided mode is prohibited and light generated in the band gap region can couple only to the radiation modes and is radiated outward. Also, the PCs fold the guided modes into the Brillouin zone boundaries, allowing phase matching to the radiation modes that lie above the air light line. ${ }^{24}$ The guided modes that phase match with the radiation modes escape eventually as Bragg scattered light. The midgap frequency of the PBG of triangular PCs of air hole in GaN with dielectric constant $\varepsilon=8.9$ is estimated to be around the normalized frequency $(a / \lambda)$ of $0.5 .^{25}$ In AlN a periodicity of less than $104 \mathrm{~nm}$ is required to tune the PBG to the emission wavelength in deep UV $(\sim 208 \mathrm{~nm})$ region. The smallest normalized frequency of our structure is 0.72 for emission wavelength at $208 \mathrm{~nm}$, which implies that photon modes in our structure lie above the cutoff frequency of guided modes. We therefore believe that the PL enhancement in our AlN PCs is mainly due to coupling of leaky modes above the cutoff frequency of the Bloch guided modes. Another factor is that the light cannot escape from AlN epilayers due to the unique polarization property. With PC forma- tion, much of the $\mathbf{E} / / c$ emission component can be extracted from the epilayers.

In summary, PCs with feature size down to $75 \mathrm{~nm}$ have been fabricated on AlN epilayers. The optical properties were studied with deep UV PL spectroscopy. PC formation produced an enhancement factor of about 20 in the band edge PL emission intensity over unpatterned AlN epilayer at $208 \mathrm{~nm}$ for $a / d=180 / 90 \mathrm{~nm}$. It was found that the PL enhancement factor increases with decreasing lattice constant. The emission peak energy was observed to decrease with decreasing lattice constant possibly due to the release of compressive strain in AlN epilayers upon PC formation.

This research is supported by grants from NSF, ARO, and DAPRA.

${ }^{1}$ J. Li, K. B. Nam, M. L. Nakarmi, J. Li, J. Y. Lin, H. X. Jiang, P. Carrier, and S. H. Wei, Appl. Phys. Lett. 83, 5163 (2003).

${ }^{2}$ K. B. Nam, J. Li, M. L. Nakarmi, J. Y. Lin, and H. X. Jiang, Appl. Phys. Lett. 84, 5264 (2004)

${ }^{3}$ W. B. Joyce, R. J. Bachrach, R. W. Dixon, and D. A. Sealer, J. Appl. Phys. 45, 2229 (1974).

${ }^{4}$ I. Schnitzer, E. Yablonovitch, C. Caneau, T. J. Gmitter, and A. Scherer, Appl. Phys. Lett. 63, 2174 (1993).

${ }^{5}$ R. Windisch, C. Rooman, S. Meinlschmidt, P. Kiesel, D. Zipperer, G. H. Döhler, B. Dutta, M. Kuijk, G. Borghs, and P. Heremans, Appl. Phys. Lett. 79, 2315 (2001).

${ }^{6}$ T. Fujii, Y. Gao, R. Sharma, E. L. Hu, S. P. DenBaars, and S. Nakamura, Appl. Phys. Lett. 84, 855 (2004).

${ }^{7}$ S. X. Jin, J. Li, J. Y. Lin, and H. X. Jiang, Appl. Phys. Lett. 77, 3236 (2000).

${ }^{8}$ E. Yablonovitch, Phys. Rev. Lett. 58, 2059 (1987).

${ }^{9}$ M. Boroditsky, R. Vrijen, T. F. Krauss, R. Coccioli, R. Bhat, and E. Yablonovitch, J. Lightwave Technol. 17, 2096 (1999).

${ }^{10}$ H. Y. Ryu, Y. H. Lee, R. L. Sellin, and D. Bimberg, Appl. Phys. Lett. 79 3573 (2001)

${ }^{11}$ A. A. Erchak, D. J. Ripin, S. Fan, P. Rakich, J. D. Joannopoulos, E. P. Ippen, G. S. Petrich, and L. A. Kolodziejski, Appl. Phys. Lett. 78, 563 (2001).

${ }^{12}$ T. N. Oder, J. Shakya, J. Y. Lin, and H. X. Jiang, Appl. Phys. Lett. 83 1231 (2003)

${ }^{13}$ T. N. Oder, H. S. Kim, J. Y. Lin, and H. X. Jiang, Appl. Phys. Lett. 84, 466 (2004).

${ }^{14}$ J. J. Wierer, M. R. Krames, J. E. Epler, N. F. Gardner, M. G. Craford, J. R. Wendt, J. A. Simmons, and M. M. Sigalas, Appl. Phys. Lett. 84, 3885 (2004).

${ }^{15}$ J. Shakya, K. H. Kim, J. Y. Lin, and H. X. Jiang, Appl. Phys. Lett. 85, 142 (2004).

${ }^{16}$ J. Shakya, J. Y. Lin, and H. X. Jiang, Appl. Phys. Lett. 85, 2104 (2004).

${ }^{17}$ Lu Chen and Arto V. Nurmikko, Appl. Phys. Lett. 85, 3663 (2004).

${ }^{18}$ A. David, C. Meier, R. Sharma, F. S. Diana, S. P. DenBaars, E. Hu, S. Nakamura, C. Weisbuch, and H. Benisty, Appl. Phys. Lett. 87, 101107 (2005).

${ }^{19}$ D.-Ho Kim, C.-O. Cho, Y.-G. Roh, H. Jeon, Y. S. Park, J. Cho, J. S. Im, C. Sone, Y. Park, Won Jun Choi, and Q.-Han Park, Appl. Phys. Lett. 87, 203508 (2005).

${ }^{20}$ M. Khizar, Z. Y. Fan, K. H. Kim, J. Y. Lin, and H. X. Jiang, Appl. Phys. Lett. 86, 173504 (2005).

${ }^{21}$ http://www.phys.ksu.edu/area/GaNgroup

${ }^{22}$ M. Bickermann, B. M. Epelbaum, P. Heimann, Z. G. Herro, and A. Winnacker, Appl. Phys. Lett. 86, 131904 (2005).

${ }^{23}$ Michael Dudley (private communication).

${ }^{24}$ V. N. Astratov, I. S. Culashaw, R. M. Stevenson, D. M. Whittaker, M. S. Skolnick, T. F. Krauss, and R. M. De La Rue, J. Lightwave Technol. 17, 2050 (1999).

${ }^{25}$ A. Barra, D. Cassagne, and C. Jouanin, Phys. Status Solidi A 176, 747 (1999). 